\title{
ESTUDO DA ZONA AFETADA PELO CALOR DE AÇO API 5L X100 COM SIMULAÇÃO COMPUTACIONAL E FİSICA*
}

\author{
Victor Hugo Pereira Moraes e Oliveira ${ }^{1}$ \\ João da Cruz Payão Filho² \\ Izabela Ferreira Girão ${ }^{3}$ \\ Ismael Ribeiro Ramos ${ }^{4}$
}

\section{Resumo}

A indústria petrolífera demanda tubos com resistência mecânica e tenacidade à fratura cada vez maiores. Estas propriedades, determinadas pela microestrutura, são modificadas completamente na soldagem. Este trabalho avaliou a formação de constituinte MA na ZAC de grãos grosseiros, reaquecidas ou não intercriticamente, de junta de topo circunferencial para tubos de aço API 5L X100 desenvolvido para águas profundas. Simularam-se com elementos finitos e em sistema Gleeble ciclos térmicos de soldagem com diferentes aportes de calor para processo FCAW. Foram feitas análises com microscopia ótica e eletrônica de varredura, além de medidas de microdureza. A morfologia do constituinte MA, cuja fração foi maior nas simulações com o maior e o menor aporte de calor e mínima para o aporte de $2,0 \mathrm{~kJ} / \mathrm{mm}$, foi muito alterada pela temperatura máxima simulada. A simulação da soldagem mostrou ser bastante complexa, e vários fatores críticos que afetam a tenacidade das juntas soldadas foram analisados por meio de simulação computacional.

Palavras-chave: Aço API 5L X100; Zona Afetada pelo Calor; Simulação de Soldagem; Constituinte MA.

\section{HEAT AFFECTED ZONE STUDY OF API 5L X100 STEEL THROUGH COMPUTATIONAL AND THERMAL WELDING SIMULATION}

\section{Abstract}

The oil\&gas industry requires pipes with increasing mechanical strength and fracture toughness. These properties, determined by the microstructure, are completely modified during the welding. This work evaluated the MA constituent formation in the coarse grain HAZ, reheated or not intercritically, of girth welded joints of API 5L X100 steel pipes, which are developed for deep water applications. Thermal cycles for different FCAW heat inputs were simulated with finite elements method and in Gleeble machine. Optical and SEM microscopy were realized, and microhardness testing was performed too. The MA constituent morphology, which fraction was higher in welds made with the highest and lowest heat input and minimal for 2.0 $\mathrm{kJ} / \mathrm{mm}$, was greatly modified by the simulated peak temperature. The welding simulation showed to be quite complex, and several critical factors that affect the toughness of welded joints were analyzed through computational simulation.

Keywords: API 5L X100 steel; Heat Affected Zone; Weld Simulation; MA Constituent.

1 Engo Metalúrgico, Mestrando, Pesquisador, Progr. de Enga Metalúrgica e Materiais, UFRJ, Rio de Janeiro, RJ, Brasil.

2 Engo Metalúrgico, Dr.-Ing., Professor, Progr. de Enga Metalurgia e Materiais, UFRJ, Rio de Janeiro, RJ, Brasil.

3 Enga de Materiais, Mestranda, Pesquisadora, Tenaris do Brasil, Pindamonhangaba, SP, Brasil.

4 Graduando, Depto. de Enga Metalúrgica e Materiais, UFRJ, Rio de Janeiro, RJ, Brasil. 


\section{INTRODUÇÃO}

Com a contínua evolução da demanda por petróleo e gás, hoje se faz necessário a exploração de reservatórios de petróleo de menor acessibilidade, em meios mais severos e mais afastados dos polos de processamento e uso, tornando a operação de transporte um fator crítico na indústria. Essa situação se reforça dentro do cenário do pré-sal brasileiro. Assim, existe um apelo constante para que os aços usados em dutos apresentem resistência mecânica, soldabilidade, tenacidade e resistência à corrosão cada vez maior. Os aços microligados de alta resistência, que vem sido desenvolvidos para aplicação nesses dutos, apresentam excelentes propriedades graças a melhoras no refino do aço, adição controlada de microligantes $(\mathrm{Nb}, \mathrm{V}, \mathrm{Ti})$, menores teores de carbono, laminação a quente controlada e resfriamento acelerado. Contudo, esses aços se mostram sensíveis a grandes alterações microestruturas quando expostos a ciclos térmicos de soldagem, especialmente em condições de solda multi-passe [1], com diminuição proeminente de tenacidade e variações locais importantes de dureza.

Efetivamente, a variação de microestrutura e propriedades na zona afetada pelo calor (ZAC) em função da temperatura de pico e da taxa de resfriamento é objeto de diversos trabalhos [1-10]. Estes indicam que a região mais próxima à linha de fusão, nomeada usualmente de região de grão grosseiros (ZAC GG) e cuja temperatura chega a patamares próximos de $1350^{\circ} \mathrm{C}$, é a região de menor tenacidade da junta, sobretudo quando exposta a um segundo aquecimento a temperaturas intercríticas (ZAC GG-IC) [1 - 4]. Essa perda de tenacidade é associada a uma microestrutura mais grosseira e à presença de ilhas de martensita não revenida e austenita retida enriquecidas em carbono, denominadas de constituinte MA [2]. Esse constituinte apresenta alta dureza e uma concentração local de tensão em sua interface com a matriz, propiciando o início de trincas [4]. É importante ressaltar que o volume dessas regiões na junta é pequeno, mas seu efeito na tenacidade geral pode ser catastrófico, a depender de diversos fatores, como: a fração, composição química e morfologia do constituinte MA, a microestrutura da matriz e o volume total da zona localizada de alta fragilidade na junta [5].

Face aos efeitos exercidos pelo constituinte MA, vários estudos avaliaram a influência dos parâmetros de soldagem na sua formação e sua relação com a tenacidade. Matsuda F. [6] coletou dados que apontam para um aumento da fração e do tamanho do MA com a diminuição da taxa de resfriamento, tendo observado uma redução quase linear da tenacidade frente com o aumento da fração de MA. Matsuda F. [6] também relata maior propensão à formação de MA em aços com maior teor de $\mathrm{C}, \mathrm{N}, \mathrm{B}, \mathrm{Mo}, \mathrm{Nb}$ e $\mathrm{V}$. Essa formação está relacionada à segregação de carbono na frente de transformação $\mathrm{Y} \rightarrow \mathrm{a}$, que estabiliza $\mathrm{y}$ e aumenta sua temperabilidade [5]. É visto que o teor de carbono nesse constituinte pode chegar facilmente a $1,1 \%$, mesmo para uma matriz com $0,06 \%$ em peso de carbono [7].

Além da dependência com a taxa de resfriamento (associada ao aporte de calor e preaquecimento da soldagem), Li X. [8] avaliou o efeito da temperatura de pico intercrítica na tenacidade da ZAC, observando pior comportamento em amostras simuladas a temperaturas intercríticas mais baixas, situação onde o MA teve formato alongado e contínuo nos contornos de austenita prévia. Sobre a morfologia, Li e Baker [9] reportaram quatro formatos: ilhas massivas usualmente vistas em pontos de interseção tripla de grãos, feixes finos e alongados entre placas de bainita, 
partículas menores que agregam MA com carbonetos internos e feixes conectados ao longo do contorno da austenita prévia. Este último formato levaria à maior fragilidade. Li X. [9] observou ainda que o tamanho de grão herdado do primeiro ciclo térmico de grãos grosseiros tem efeito significativo na tenacidade e que o refino deste poderia reduzir a queda de tenacidade na ZAC GG-IC.

Dentro desta problemática, avaliou-se neste trabalho o efeito do aporte de calor e da temperatura de pico intercrítica em um tubo de aço API $5 \mathrm{~L}$ X100, buscando parâmetros de soldagem que minimizassem a formação de zonas localizadas de alta fragilidade. À diferença da bibliografia geral sobre o tema, neste trabalho utilizou-se uma abordagem mais embasada na simulação computacional (métodos de elementos finitos/Sysweld e monte carlo/JMATPRO), ferramenta capaz agilizar a otimização de parâmetros de soldagem, evidenciando o efeito de cada um desses parâmetros e das características do material de base a um custo bem reduzido. Além disso, a simulação computacional vem permitindo incorporar os fenômenos vistos em microescala ao comportamento macro dos componentes (no caso da junta soldada), fornecendo assim uma compreensão multi-escala do comportamento do componente em sua fabricação e operação, para um custo computacional cada vez menor. Acredita-se então que o futuro do desenvolvimento das técnicas de produção e processamento de materiais será permeado, invariavelmente, pela evolução desses métodos de simulação computacional multi-escala, reforçando a importância dos esforços deste trabalho.

\section{MATERIAIS E MÉTODOS}

Se procedeu com a simulação computacional de soldagem por método de elementos finitos utilizando o software comercial Sysweld. Foi considerada uma junta de topo circunferencial, soldada por processo arame tubular (FCAW) em tubo com 20" de diâmetro externo, 0,625" de espessura e composição química conforme a Tabela 1.

Tabela 1. Composição química do aço API $5 \mathrm{~L}$ X100

\begin{tabular}{|c|c|c|c|c|c|c|}
\hline$\% \mathbf{C}$ & $\% \mathbf{M n}$ & $\% \mathbf{N b}+\mathbf{T i}+\mathbf{V}$ & $\% \mathbf{S}$ & $\% \mathbf{P}$ & $\mathbf{P}_{\mathrm{cm}}$ & $\mathbf{C}_{\mathbf{E}}$ \\
\hline 0,07 & 1,88 & 0,023 & 0,001 & 0,013 & 0,2 & 0,49 \\
\hline
\end{tabular}

Os parâmetros de soldagem da simulação foram baseados em valores de uma junta previamente soldada, cujas macrografias permitiram a modelagem 3D dos cordões de solda no software. Na Tabela 2 resumem-se as informações que deram base aos dados de entrada da simulação computacional.

Tabela 2. Macrografia e parâmetros gerais de soldagem real que serviram de base para simulação

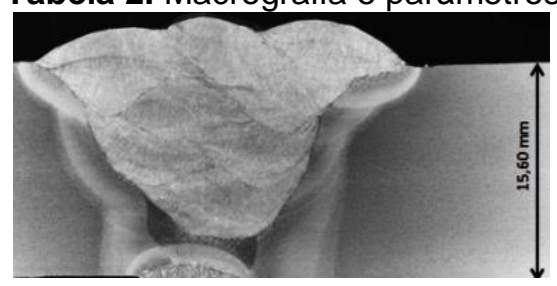

\begin{tabular}{|c|}
\hline Preaquecimento: $150-250^{\circ} \mathrm{C}$ \\
\hline Temperatura Interpasse: $100-250^{\circ} \mathrm{C}$ \\
\hline Corrente: $150-180 \mathrm{~A}$ \\
\hline Voltagem: $15-22 \mathrm{~V}$ \\
\hline Velocidade de Soldagem: $2,4-2,8 \mathrm{~mm} / \mathrm{s}$ \\
\hline Aporte médio de Calor: $1,1 \mathrm{~kJ} / \mathrm{mm}$ \\
\hline Fator de eficiência térmica: 0,8 \\
\hline
\end{tabular}

Com intuito de estudar o efeito do aporte térmico na microestrutura, variaram-se na simulação alguns parâmetros de soldagem, obtendo quatro aportes de calor representativos da aplicação em campo: 0,5, 1,5, 2,0 e 3,0 kJ/mm. Na Tabela 3 esses parâmetros são sumarizados por aporte de calor. 
Tabela 3. Parâmetros de soldagem utilizados na simulação computacional de soldagem

\begin{tabular}{|c|c|c|c|}
\hline Aporte de Calor [kJ/mm] & Tensão [V] & Corrente [A] & Velocidade de Soldagem [mm/s] \\
\hline 0,5 & 22 & 120 & 4,00 \\
\hline 1,5 & 22 & 160 & 1,90 \\
\hline 2,0 & 22 & 180 & 1,55 \\
\hline 3,0 & 22 & 200 & 1,20 \\
\hline
\end{tabular}

Com esses parâmetros foram simuladas sete condições de soldagem: primeiramente, três representando um passe único, logo após a raiz (conhecido como passe-quente), com aportes de 0,5, 1,5 e $3,0 \mathrm{~kJ} / \mathrm{mm}$; em seguida, mais quatro condições, representando os passes múltiplos de enchimento, com aporte de 0,5, 1,5, 2,0 e 3,0 kJ/mm. Extraiu-se das simulações os ciclos térmicos de pontos que se enquadrassem o mais próximo possível de um pico a $1350^{\circ} \mathrm{C}$, representando a zona afetada pelo calor de grãos grosseiros (ZAC GG), e dois picos a $1350^{\circ} \mathrm{C}$ e $850^{\circ} \mathrm{C}$, representando a região de grãos grosseiros reaquecida à temperatura intercrítica (ZAC GG-IC). A exatidão das temperaturas de pico selecionadas é dependente do refinamento da malha na região de interesse, com uma tolerância de até $25^{\circ} \mathrm{C}$ para o pico de $1350^{\circ} \mathrm{C}$ e $30^{\circ} \mathrm{C}$ para o pico de $850^{\circ} \mathrm{C}$. Importante notar que, para fins de simplificação, os ciclos térmicos subcríticos de passes intermediários do enchimento foram cortados, mantendo-se apenas a temperatura interpasse, resultando em uma condição de passe duplo.

A simulação computacional também permitiu prever as fases e a dureza das regiões de interesse (ZAC GG e GG-IC). A extensão em comprimento e as sobreposições de áreas da ZAC também foram medidas. Essa previsão é feita inserindo o diagrama de transformação em resfriamento contínuo (TRC) do material no software de simulação Sysweld. É através dos modelos de cinética de transformação JohnsonMehl-Avrami/Leblond (difusional) e Koistinen-Marburger (martensítica) [10] que o software estima a fração transformada na ciclagem térmica. Com base na fração de fases, composição química e equações empíricas (Blondeu R.) [10], estima-se a dureza. Utilizaram-se dois diagramas TRC: um retirado do trabalho de Liu Y. [11], levantado empiricamente para soldagem em material de composição similar, e outro desenvolvido através de simulação computacional em software comercial JMATPRO, utilizando a composição química analisada do material do estudo. Os resultados obtidos com os diferentes diagramas foram então comparados. Os diagramas TRC são apresentados na Figura 1.
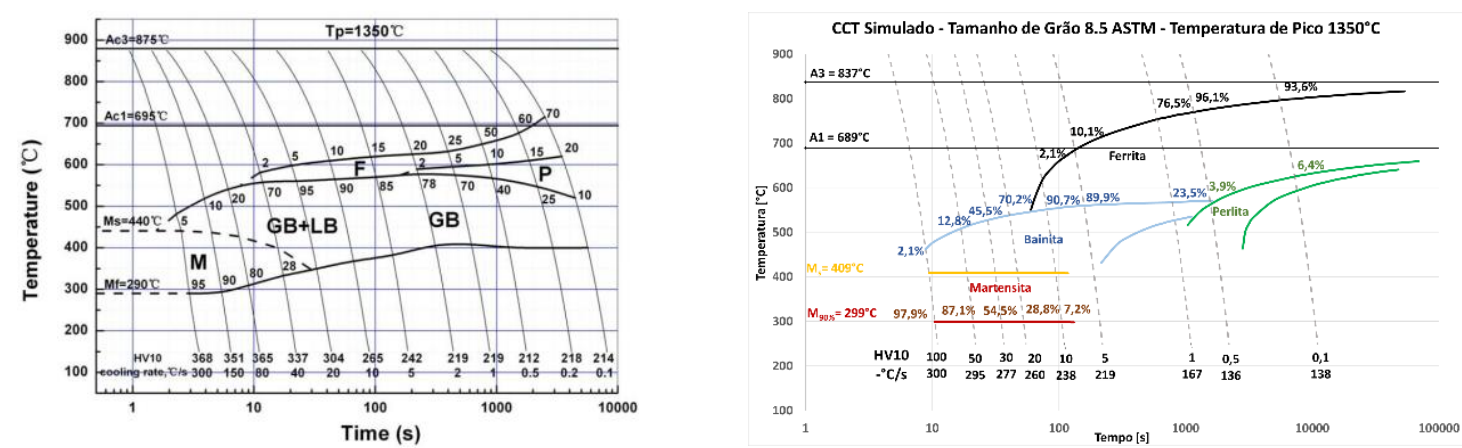

Figura 1. Diagramas TRC: à esquerda retirado de Liu Y.[11], à direita simulado em software JMATPRO.

Após a simulação computacional, foram retirados corpos de prova de dimensional 10 $\mathrm{mm} \times 10 \mathrm{~mm} \times 85 \mathrm{~mm}$ de um tubo API 5L X100, com o lado de maior comprimento do corpo de prova paralelo à direção de laminação e eixo axial do tubo. Estes corpos 
de prova foram então levados ao sistema Gleeble 3800 e submetidos à simulação térmica conforme dados da simulação computacional. A temperatura real no corpo de prova foi monitorada e controlada através de termopares de platina, instalados no centro do espécime. Na Figura 2 se mostra a montagem do ensaio. Para os ensaios da ZAC GG-IC, utilizou-se um segundo termopar a $3 \mathrm{~mm}$ do primeiro.
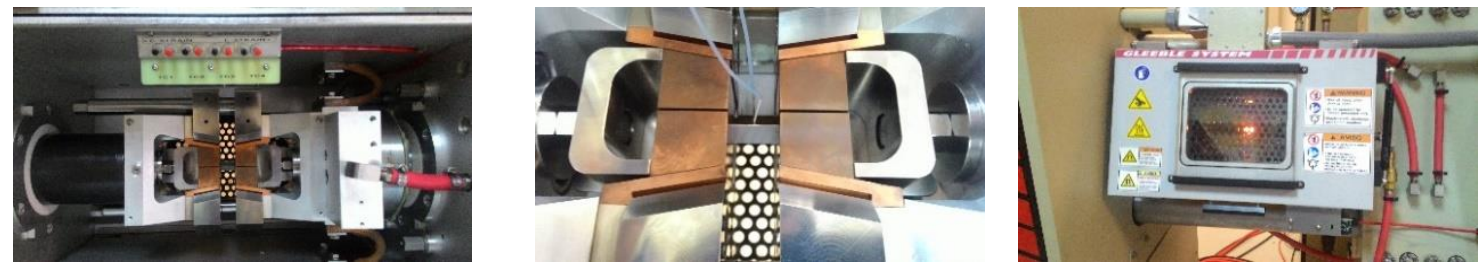

Figura 2. Sistema Gleeble 3800 montado para simulação térmica de ZAC

Antes dos ensaios de simulação térmica de ZAC GG-IC, realizaram-se ensaios de dilatometria para definir as temperaturas $A 1$ e $A 3$ do material, permitindo a seleção acurada da temperatura de pico intercrítica para o segundo passe. Tendo em vista o possível efeito da taxa de aquecimento sobre essas temperaturas características, realizaram-se ensaios com alta $\left(200^{\circ} \mathrm{C} / \mathrm{s}\right)$ e baixa $\left(0,5^{\circ} \mathrm{C} / \mathrm{s}\right)$ taxa de aquecimento, cada uma com três amostras. Além da medição experimental, também foi simulado no software JMATPRO o efeito da taxa de aquecimento nessas temperaturas, através do diagrama tempo temperatura austenitização (TTA).

Após a simulação térmica, corpos de prova foram reservados para ensaios de impacto Charpy e uma amostra de cada uma das sete condições de ciclo térmico foi retirada para estudo microestrutural. Essas amostras tiveram a face de maior área preparada, sendo lixadas até lixa de granulometria de 1200, polidas em pasta de diamante de até $1 \mu \mathrm{m}$ e depois atacadas por imersão em nital $2 \%$ por aproximadamente 11 segundos para revelar sua microestrutura geral. Observou-se a microestrutura atacada em microscópio ótico ZEISS Imager M2m (até aumentos de 1000x) e também em microscópio eletrônico de varredura JEOL (até aumentos de 5000x). As mesmas amostras foram novamente lixadas e polidas para passarem por um novo ataque, desta vez eletrolítico e com duas etapas. A primeira etapa em solução de $5 \mathrm{~g}$ EDTA e $0,5 \mathrm{~g}$ de fluoreto de sódio em $100 \mathrm{ml}$ de água destilada, sob potencial de 5,1V por cerca de 11 segundos. Na segunda etapa, após lavar a amostra com álcool, usou-se uma solução com $5 \mathrm{~g}$ de ácido pícrico e $25 \mathrm{~g}$ de hidróxido de sódio em $10 \mathrm{ml}$ de água destilada, sob potencial de 7,1V por 135 segundos. Esse procedimento eletrolítico permitiu atacar preferencialmente grãos de ferrita (bainita) e carbonetos, respectivamente, deixando em alto relevo o constituinte MA, permitindo contraste ao MEV em elétron secundário.

A partir das imagens de microscopia ótica em aumento de 1000x com ataque de nital $2 \%$, avaliou-se o tamanho de grãos da austenita prévia das amostras de ZAC GG. Foi utilizado método de interceptos, conforme norma ASTM 112. Nas imagens de MEV em aumento de 1000x com ataque eletrolítico duplo foi feito um tratamento de imagem com o software ImageJ, de forma a permitir a quantificação do percentual e morfologia do constituinte MA nas sete condições de ciclo térmico simuladas. Após as análises metalográficas, as amostras foram submetidas a ensaio de microdureza Vickers, com carga $500 \mathrm{~g}$. Foram realizadas 15 (quinze) indentações na região central do corpo de prova simulado utilizando microdurômetro Indentec modelo ZHV/M/2009. 


\section{RESULTADOS E DISCUSSÃO}

\subsection{Ensaios de Dilatometria}

O resultado médio da dilatometria e também os valores na simulação com software JMATPRO são apresentados na Tabela 4. Esses resultados confirmam o regime intercrítico à temperatura de $850^{\circ} \mathrm{C}$ e o efeito expressivo da alta taxa de aquecimento, fator comum à soldagem, no aumento de A1 e A3. Ressalta-se que a simulação obteve resultados bem próximos aos de ensaio para $A 1$, mas foi menos acurada para A3, subestimando as temperaturas.

Tabela 4. Resultados médios de ensaios de dilatometria e valores obtidos com JMATPRO (TTA)

\begin{tabular}{|c|c|c|c|c|}
\hline \multirow{2}{*}{ Método } & \multicolumn{2}{|c|}{ Dilatometria } & \multicolumn{2}{c|}{ Simulação } \\
\cline { 2 - 5 } & $\mathbf{0 , 5 ^ { \circ }} \mathbf{C} / \mathbf{s}$ & $\mathbf{2 0 0}{ }^{\circ} \mathbf{C} / \mathbf{s}$ & $\mathbf{1}^{\circ} \mathbf{C} / \mathbf{s}$ & $\mathbf{1 0 0} \mathbf{C} / \mathbf{s}$ \\
\hline $\left.\mathrm{A} 1{ }^{\circ} \mathrm{C}\right]$ & 694 & 740 & 688 & 741 \\
\hline $\left.\mathrm{A} 3{ }^{\circ} \mathrm{C}\right]$ & 908 & 956 & 836 & 875 \\
\hline
\end{tabular}

\subsection{Simulação Computacional em Elementos Finitos}

$\mathrm{Na}$ Figura 3 ilustra-se o resultado da simulação de soldagem com aporte de $1,5 \mathrm{~kJ} / \mathrm{mm}$, com uma imagem de temperatura de pico máximo em cada ponto da malha e com a curva ciclo térmico completa de um ponto da ZAC GG-IC.
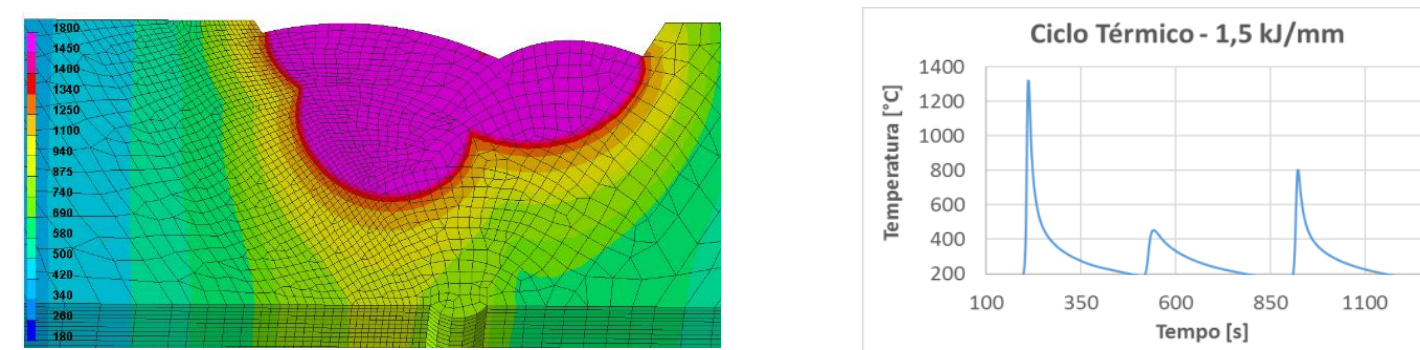

Figura 3. Simulação computacional: isotermas máximas e ciclo térmico ZAC GG-IC

$\mathrm{Na}$ Tabela 5 sumarizam-se os principais parâmetros dos ciclos térmicos de soldagem resultantes da simulação. De modo geral, o tempo e a taxa de resfriamento médio entre $800^{\circ} \mathrm{C}$ e $500^{\circ} \mathrm{C}$ seguiram a tendência esperada e apresentaram concordância com valores encontrados na bibliografia [12]. Chama-se atenção para o fato de que a variação de altura dos passes e do volume preenchido do chanfro entre as condições tem efeito pequeno, porém, relevante nas taxas de resfriamento. Os aportes de 2,0 e $3,0 \mathrm{~kJ} / \mathrm{mm}$ apresentaram um resultado bem próximo, inclusive, com maior taxa de resfriamento para o maior aporte de calor, no caso do primeiro pico na condição de passe duplo. Essa discrepância pode ser justificada pelo fato de que a geometria dos cordões foi balizada em macrografias de uma solda de aporte de calor médio de $1,1 \mathrm{~kJ} / \mathrm{mm}$ e depois extrapolada para os demais aportes de calor, provavelmente com maior taxa de erro para o aporte mais distantes. Este ponto chama atenção de como é importante a utilização de macrografias de soldas reais na parametrização da simulação computacional.

A extensão de ZAC em função de isotermas de temperatura máxima alcançada em cada ponto é apresentada na Tabela 6 , considerando as regiões de grãos grosseiros (GG), grãos finos (GF), intercrítica (IC) e subcrítica (SC), para os diferentes aportes. Essa análise se restringiu à condição de passe único, considerando sua replicabilidade para passes múltiplos. 
Tabela 5. Principais informações dos ciclos térmicos de soldagem resultantes da simulação

\begin{tabular}{|c|c|c|c|c|c|c|c|c|}
\hline \multirow{2}{*}{$\begin{array}{c}\text { Aporte de } \\
\text { Calor } \\
{[\mathrm{kJ} / \mathrm{mm}]}\end{array}$} & \multirow[b]{2}{*}{$\begin{array}{l}\text { Condição } \\
\text { de passe }\end{array}$} & \multirow{2}{*}{$\begin{array}{c}\text { Espessura } \\
\text { preenchida } \\
\text { antes do } 1^{\circ} \\
\text { passe }\end{array}$} & \multicolumn{3}{|c|}{$1^{\circ}$ passe } & \multicolumn{3}{|c|}{$2^{\circ}$ passe } \\
\hline & & & $\begin{array}{l}\text { Tpico } \\
{\left[{ }^{\circ} \mathrm{C}\right]}\end{array}$ & $\begin{array}{l}t_{8 / 5} \\
{[s]}\end{array}$ & $\begin{array}{c}\text { Resfriamento } \\
8 / 5\left[{ }^{\circ} \mathrm{C} / \mathrm{s}\right]\end{array}$ & $\begin{array}{c}\text { Tpico } \\
{\left[{ }^{\circ} \mathrm{C}\right]}\end{array}$ & $\begin{array}{l}t_{8 / 5} \\
{[s]}\end{array}$ & $\begin{array}{l}\text { Resfriamento } \\
8 / 5\left[{ }^{\circ} \mathrm{C} / \mathrm{s}\right]\end{array}$ \\
\hline 0,5 & Único & 2,1 & 1345,9 & 5,5 & 54,5 & - & - & - \\
\hline 1,5 & Único & 2,1 & 1349,6 & 17,7 & 16,9 & - & - & - \\
\hline 3,0 & Único & 2,1 & 1363,8 & 38,4 & 8,1 & 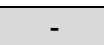 & 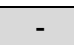 & - \\
\hline 0,5 & Duplo & 3,6 & 1339,1 & 5,2 & 57,4 & 844,2 & 5,1 & 59,0 \\
\hline 1,5 & Duplo & 6,3 & 1336,5 & 21,4 & 14,0 & 847,7 & 17,2 & 17,4 \\
\hline 2,0 & Duplo & 8,0 & 1345,9 & 36,6 & 8,2 & 849,6 & 30,3 & 9,9 \\
\hline 3,0 & Duplo & 8,5 & 1363,5 & 33,1 & 9,1 & 881,6 & 33,7 & 8,9 \\
\hline
\end{tabular}

Tabela 6. Extensão da ZAC determinada por simulação computacional

\begin{tabular}{|c|c|c|c|c|}
\hline \multirow{2}{*}{$\begin{array}{c}\text { Aporte de } \\
\text { Calor }[\mathrm{kJ} / \mathrm{mm}]\end{array}$} & \multicolumn{4}{|c|}{ Extensão da ZAC [mm] } \\
\hline & $\begin{array}{c}\text { ZAC-GG }\left(1450^{\circ} \mathrm{C}-\right. \\
\left.1250^{\circ} \mathrm{C}\right)\end{array}$ & $\begin{array}{c}\text { ZAC-GF }\left(1250^{\circ} \mathrm{C}\right. \\
\left.-940^{\circ} \mathrm{C}\right)\end{array}$ & $\begin{array}{c}\text { ZAC-IC }\left(940^{\circ} \mathrm{C}-\right. \\
\left.740^{\circ} \mathrm{C}\right)\end{array}$ & $\begin{array}{c}\text { ZAC-SC }\left(740^{\circ} \mathrm{C}-\right. \\
\left.500^{\circ} \mathrm{C}\right)\end{array}$ \\
\hline 0,5 & 0,6 & 1,0 & 0,8 & 2,4 \\
\hline 1,5 & 1,2 & 1,9 & 1,8 & 4,8 \\
\hline 3,0 & 1,6 & 2,7 & 2,6 & 7,4 \\
\hline
\end{tabular}

Para condição de passes múltiplos, analisou-se a sobreposição de regiões do primeiro e segundo passe. Avaliaram-se as diversas regiões de sobreposição dentro da ZAC GG do primeiro passe, com foco na ZAC GG-IC, subdividida em cinco faixas de temperatura, tendo em vista o efeito da temperatura de pico do segundo passe na formação do constituinte MA e sua morfologia. Conforme discutido na introdução deste trabalho, a morfologia do constituinte MA formado a temperaturas intercríticas mais baixas seria mais prejudicial à tenacidade da junta. A Tabela 7 mostra a proporção das regiões de sobreposição para os diferentes aportes e uma imagem ilustrativa da metodologia.

Tabela 7. Estudo de sobreposição de regiões reaquecidas da ZAC GG

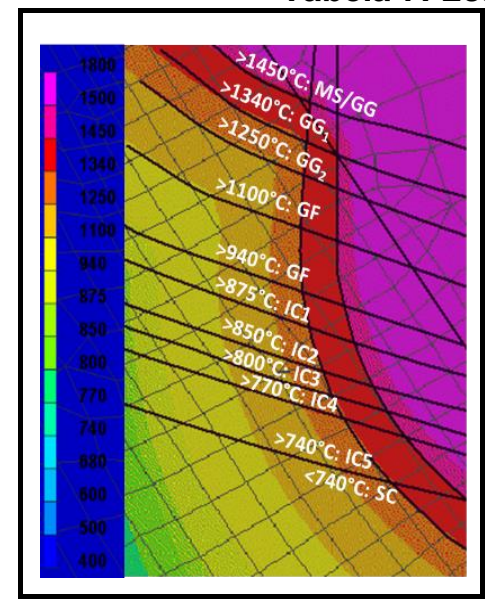

\begin{tabular}{|c|c|c|c|c|c|}
\hline \multicolumn{6}{|c|}{ Fração de sobreposição [\%] } \\
\hline \multirow{2}{*}{ Temperaturas } & \multirow{2}{*}{ Região } & \multicolumn{4}{|c|}{ Aporte de Calor $[\mathrm{kJ} / \mathrm{mm}]$} \\
\hline & & 0,5 & 1,5 & 2,0 & 3,0 \\
\hline$>1450^{\circ} \mathrm{C}$ & MS/GG & 0,8 & $\overline{1,1}$ & 3,1 & $\overline{3,9}$ \\
\hline$>1340^{\circ} \mathrm{C}$ & $\mathrm{GG}_{1}$ & 1,2 & 2,6 & 5,0 & 6,6 \\
\hline$>1250^{\circ} \mathrm{C}$ & $\mathrm{GG}_{2}$ & 2,3 & 3,3 & 4,0 & 6,1 \\
\hline$>940^{\circ} \mathrm{C}$ & GF & 12,2 & 16,7 & 21,8 & 54,9 \\
\hline$>875^{\circ} \mathrm{C}$ & $\mathrm{IC}_{1}$ & 7,2 & 9,2 & 9,1 & 24,2 \\
\hline$>850^{\circ} \mathrm{C}$ & $\mathrm{IC}_{2}$ & 9,0 & 8,9 & 2,3 & 3,9 \\
\hline$>800^{\circ} \mathrm{C}$ & $\mathrm{IC}_{3}$ & 5,3 & 5,7 & 11,6 & 0,4 \\
\hline$>770^{\circ} \mathrm{C}$ & $\mathrm{IC}_{4}$ & 5,6 & 19,3 & 43,1 & 0,0 \\
\hline$>740^{\circ} \mathrm{C}$ & $\mathrm{IC}_{5}$ & 13,8 & 34,6 & 0,0 & 0,0 \\
\hline$<740^{\circ} \mathrm{C}$ & SC & 42,6 & 0,0 & 0,0 & 0,0 \\
\hline \multicolumn{2}{|c|}{ Área total ZAC GG [mm²] } & $\overline{0,71}$ & 1,68 & 2,87 & 4,06 \\
\hline
\end{tabular}

Essa análise é essencial no estudo da perda de tenacidade (devido ao constituinte MA) para diferentes aportes de calor e sequência de passes de solda, pois o volume total da zona localizada de alta fragilidade pode influenciar mais a tenacidade da junta soldada que apenas o percentual de MA na região mais crítica de sobreposição proporcionada por um aporte de calor. Por exemplo, ainda que o aporte de calor de $1,5 \mathrm{~kJ} / \mathrm{mm}$ apresentasse menor fração de MA em sua ZAC GGIC, a sobreposição da região intercrítica inferior (abaixo de $850^{\circ} \mathrm{C}$ ) é maior que $50 \%$ da ZAC GG original, levando a um volume fragilizado maior que o aporte de calor de 
$3,0 \mathrm{~kJ} / \mathrm{mm}$. Tal análise não leva em conta a alteração de tenacidade da matriz com a variação do aporte de calor e é bem sensível à geometria dos cordões, mas ainda assim traz informação crítica quando se compara aportes de calor.

$\mathrm{Na}$ Tabela 8 apresenta-se a previsão de fases em função do diagrama TRC utilizado. Pelas observações metalográficas, os resultados para o TRC(b), simulado com a composição química analisada, ficaram mais coerentes com a realidade, baseando-se na fração de martensita e ferrita para o menor e maior aporte de calor. Apesar disso, a simulação não previu efetivamente a ocorrência de constituinte MA, conforme presença expressiva vista na microscopia. Para condição de passe único, deve-se observar que os diagramas TRC não predizem uma formação importante de MA, possivelmente por dificuldades em caracterizar o MA, no caso experimental, e em considerar nos modelos a segregação de carbono nas interfaces durante a evolução da transformação $\gamma \rightarrow \alpha$, no caso do simulado. Essa limitação é repassada diretamente a simulação por elementos finitos. Para condição de passe duplo, somase ainda o fato de que os diagramas consideram temperatura de austenitização de $1350^{\circ} \mathrm{C}$, enquanto que o pico do segundo passe austenitiza parcialmente à temperatura de $850^{\circ} \mathrm{C}$. A essa temperatura, espera-se que a austenita recémtransformada possua teores maiores de carbono, tendo maior temperabilidade. Assim esses TRCs não representam perfeitamente a ZAC reaquecida. Essas questões obviamente impactam a previsão de fases e pode justificar a imprecisão da simulação computacional. Pela bibliografia [2, 4, 6], esse enriquecimento, sem precipitação de cementita, se dá mais fortemente para condições de resfriamento mais lento, explicando o aumento de MA com o aporte de calor. Ao mesmo tempo, quanto maior a taxa de resfriamento, maior a chance da austenita enriquecida em carbono se transformar em martensita ou ser retida.

Tabela 8. Quantificação de fases resultante da simulação computacional

\begin{tabular}{|c|c|c|c|c|c|c|c|c|}
\hline \multirow{2}{*}{$\mathrm{AC}[\mathrm{kJ} / \mathrm{mm}]$} & \multirow{2}{*}{$\begin{array}{c}\text { X LF } 1^{\circ} \text { passe } \\
{[\mathrm{mm}]}\end{array}$} & \multirow{2}{*}{$\begin{array}{c}\text { X LF } 2^{\circ} \text { passe } \\
{[\mathrm{mm}]}\end{array}$} & \multicolumn{3}{|c|}{ TRC (a) } & \multicolumn{3}{|c|}{ TRC (b) } \\
\hline & & & $\% \alpha_{B}$ & $\% \alpha^{\prime}$ & $\% \alpha$ & $\% \alpha_{B}$ & $\% \alpha^{\prime}$ & $\% \alpha$ \\
\hline 0,5 & 0,3 & - & 93,34 & 5,75 & 0,90 & 50,70 & 49,29 & 0,00 \\
\hline 1,5 & 0,7 & - & 94,32 & 0,72 & 4,95 & 98,17 & 1,25 & 0,57 \\
\hline 3,0 & 0,8 & - & 87,11 & 0,71 & 12,17 & 97,02 & 0,01 & 2,97 \\
\hline 0,5 & 0,4 & 1,8 & 96,18 & 2,72 & 1,10 & 78,27 & 21,64 & 0,06 \\
\hline 1,5 & 0,6 & 3,1 & 89,70 & 0,70 & 9,60 & 98,28 & 0,70 & 1,02 \\
\hline 2,0 & 0,9 & 4,1 & 80,52 & 0,62 & 18,86 & 96,44 & 0,10 & 3,46 \\
\hline 3,0 & 1,1 & 5,1 & 81,65 & 0,57 & 17,78 & 95,23 & 0,07 & 4,63 \\
\hline $\begin{array}{l}\text { X é a distância } \\
\alpha_{B}, \alpha^{\prime} \text { e a repre } \\
\text { Percentual resic } \\
\text { (a) é o TRC reti }\end{array}$ & iografia [11] & e AC ap & & & & & & \\
\hline
\end{tabular}

\subsection{Ensaios de Microdureza}

A Figura 4 apresenta a variação da microdureza com o aporte de calor, para as condições de passe único e duplo. Complementar aos resultados experimentais, também foram incluídas na curva os valores de dureza simulados no Sysweld, considerando os TRCs previamente citados. A dureza diminui para aportes de calor maiores, tendo um pico máximo de dureza no aporte de $0,5 \mathrm{~kJ} / \mathrm{mm}$, no qual de fato se observou, tanto pela simulação computacional como pela microscopia, a presença expressiva de martensita. Para condição de passe único, a previsão de dureza pelo $\operatorname{TRC}(\mathrm{b})$ simulado se mostrou bem próxima dos valores experimentais. Contudo, essa tendência se inverteu para condição de passe duplo. Tal inversão 
deve estar relacionada aos mesmos motivos citados anteriormente para a imprecisão na previsão de MA.

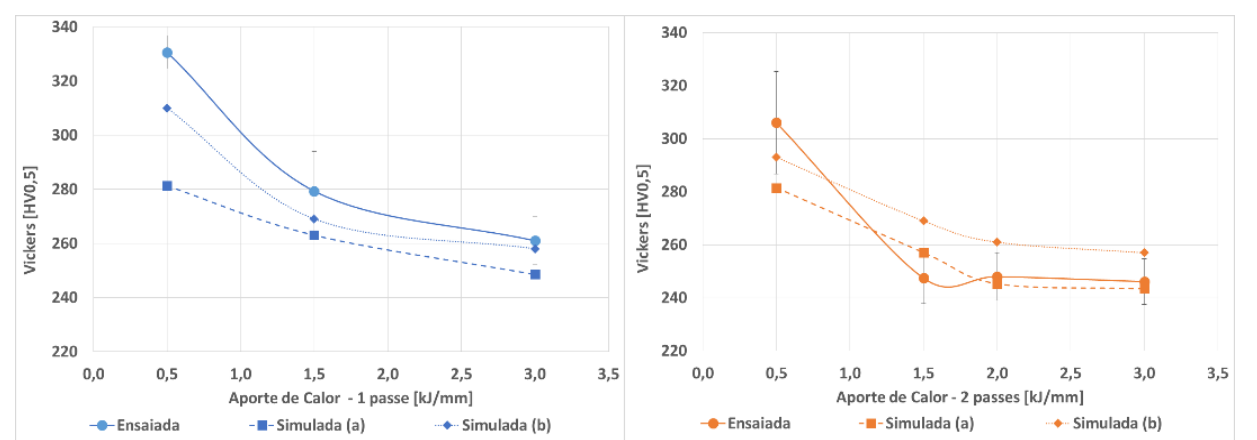

Figura 4. Gráficos de microdureza em função do aporte do calor para passe único (a esquerda) e passe duplo (a direta), com barra de erro igual a duas vezes o desvio padrão.

\subsection{Microscopia Ótica}

As micrografias óticas das amostras atacadas com nital são apresentadas nas Figuras 5 e 6, para condição de passe único e duplo, respectivamente. A amostra simulada com $0,5 \mathrm{~kJ} / \mathrm{mm}$ apresentou formação expressiva de martensita, junto a bainita inferior e superior. Já as amostras com aporte de calor de 1,5 e 3,0 kJ/mm apresentaram majoritariamente bainita superior e bainita granular, além de ferrita poligonal e bainita inferior. Para o maior aporte de calor, a presença da bainita granular foi mais preponderante e foi possível identificar ilhas mais massivas de MA. O tamanho de grão médio da austenita prévia foi de $12,7 \mu \mathrm{m}, 18,8 \mu \mathrm{m}$ e $27,9 \mu \mathrm{m}$ para os aportes de calor de 0,5, 1,5 e 3,0 kJ/mm, respectivamente.
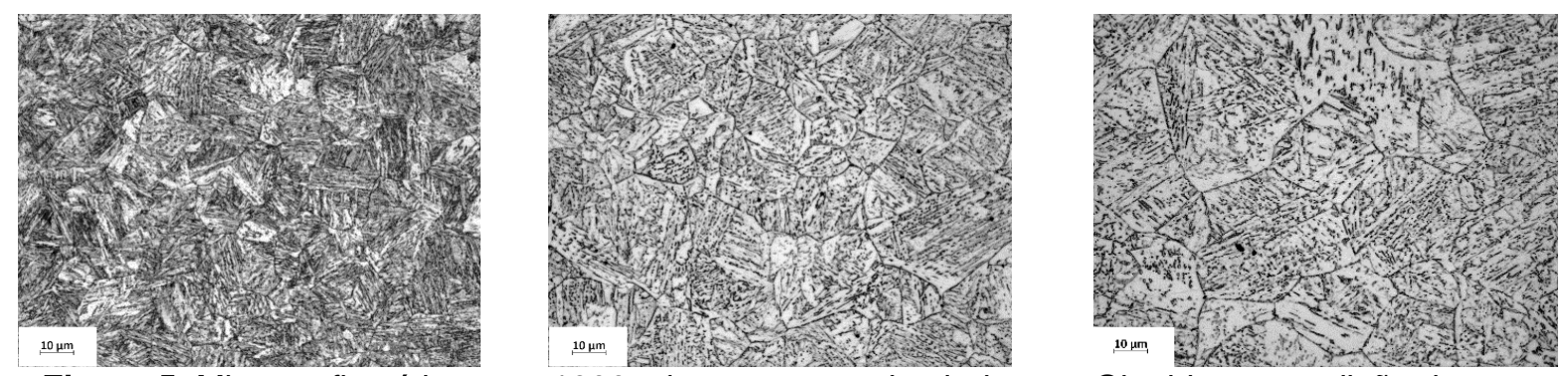

Figura 5. Micrografias óticas em 1000x das amostras simuladas em Gleeble na condição de passe único. Da esquerda para direita: aporte de calor 0,5, 1,5 e 3,0 kJ/mm

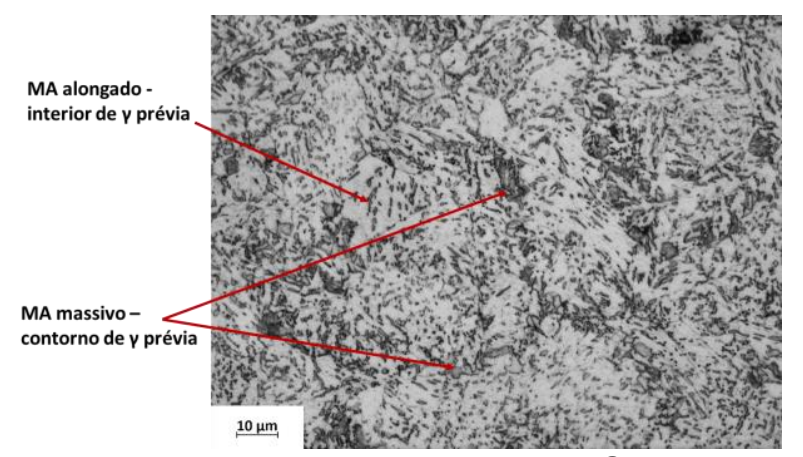

Figura 6. Micrografia ótica em 1000x de amostra simulada em Gleeble na condição de passe duplo ZAC GG-IC, para aporte de calor de $2,0 \mathrm{~kJ} / \mathrm{mm}$.

Na Figura 6 apresenta-se um exemplo de microestrutura resultante da simulação térmica em Gleeble da ZAC GG-IC. É possível discernir a presença expressiva MA 
relevado em contraste cinza escuro. Este se distribui tanto no interior do grão quanto nos contornos da austenita prévia, apesar dos contornos não serem tão facilmente identificados. É importante comentar que o corpo de prova simulado em Gleeble possui uma variação significativa de temperatura de pico alcançada em função da distância das garras de cobre, sendo a região central, onde fica o termopar de controle, a região de maior temperatura e mais próxima à programação. Assim, olhando a amostra no eixo de maior comprimento, é possível detectar uma mudança muito significativa na microestrutura da ZAC GG-IC simulada com a distância do termopar central, conforme se mostra na Figura 7. Efetivamente, um segundo termopar foi colocado a uma distância de $3 \mathrm{~mm}$ do centro e uma diferença de pelo menos $30^{\circ} \mathrm{C}$ foi detectada. A taxa de resfriamento se mostrou menos sensível a essa distância. A variação de temperatura de pico permitiu observar como a morfologia do constituinte MA se altera drasticamente com a redução de pouco mais de $30^{\circ} \mathrm{C}$, como é ilustrado na Figura 7 . Em temperaturas mais baixas o MA adquire formato alongado e contínuo ao longo dos contornos da austenita prévia. Essa observação está de acordo a bibliografia $[7,8,11]$, que chama essa morfologia de "necklace".
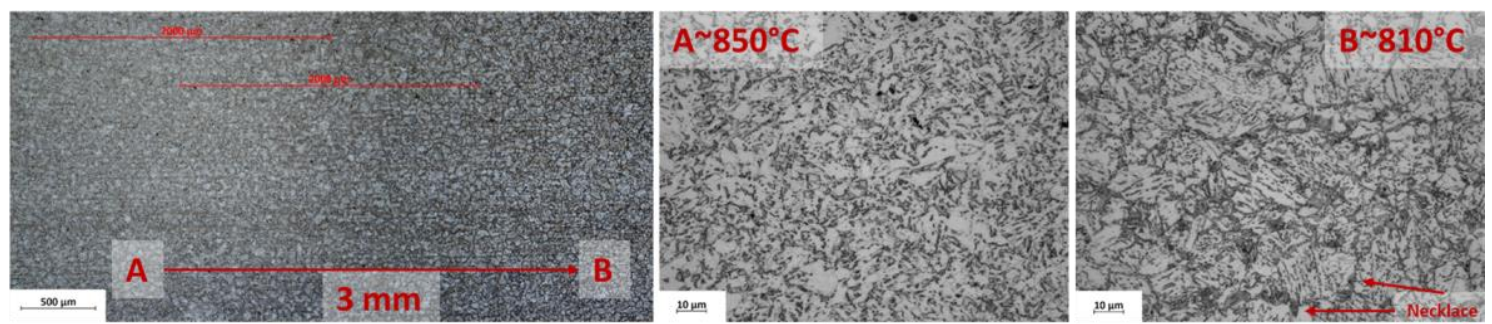

Figura 7. À esquerda mosaico de ZAC GG-IC $1,5 \mathrm{~kJ} / \mathrm{mm}$ em $50 x$ mostrando variação de microestrutura no sentido axial. Ponto $\mathrm{A}$ : região logo abaixo do termopar que atingiu $\sim 850^{\circ} \mathrm{C}$. Ponto $\mathrm{B}$ : região a $3 \mathrm{~mm}$ do termopar que atingiu $\sim 810^{\circ} \mathrm{C}$

\subsection{Microscopia Eletrônica de Varredura}

As Figuras 8 e 9 mostram o constituinte MA em contraste cinza claro, revelado pelo ataque eletrolítico, nas amostras simuladas em Gleeble para ZAC GG e ZAC GG-IC, respectivamente, em MEV.
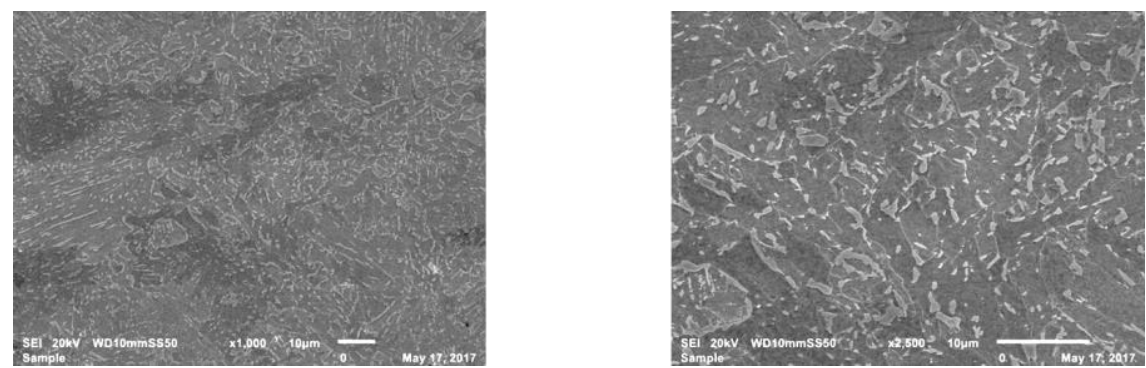

Figura 8. ZAC GG aporte de calor $3,0 \mathrm{~kJ} / \mathrm{mm}$, aumentos de 1000x e 5000x, ataque eletrolítico

Na Figura 9, vemos novamente a modificação drástica da morfologia do constituinte MA em função da temperatura de pico alcançada para uma variação de quase $40^{\circ} \mathrm{C}$. O constituinte MA da região central das amostras simuladas em Gleeble foi quantificado a partir das imagens em MEV. Os resultados desta quantificação são resumidos na Figura 10. A fração de MA mostrou tendência de aumento para os extremos de aporte de calor e valor mínimo para o aporte de $2,0 \mathrm{~kJ} / \mathrm{mm}$. O mesmo comportamento foi observado para mediana da área individual de cada partícula de MA. A razão de aspecto apresentou tendência inversa. Isso indica que para os 
aportes de calor de 0,5 e $3,0 \mathrm{~kJ} / \mathrm{mm}$ as ilhas de MA são maiores e mais massivas, além de ocorrer em maior fração. Zhuang $C$ [1] achou quantidade similar de MA em seu trabalho.
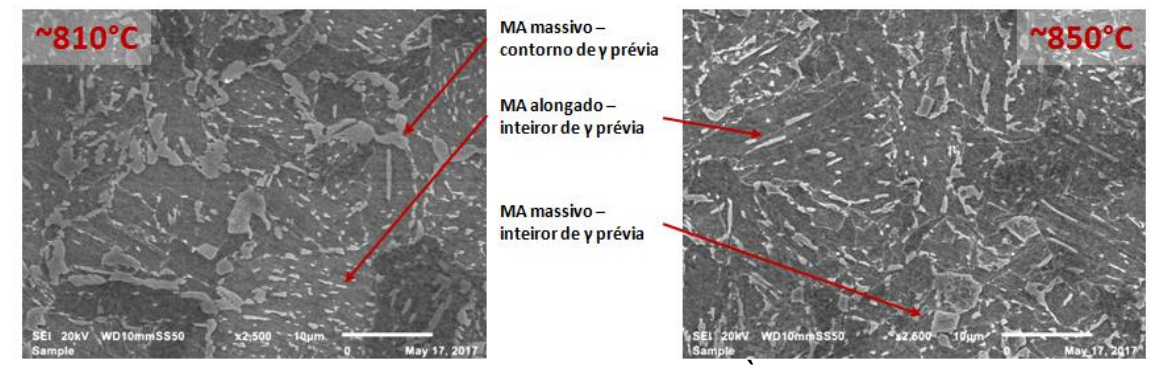

Figura 9. ZAC GG-IC $(1,5 \mathrm{~kJ} / \mathrm{mm}) 2500 x$, ataque eletrolítico. À direta: região logo abaixo do termopar, temperatura de pico $\sim 850^{\circ} \mathrm{C}$. À esquerda: região a $3 \mathrm{~mm}$ do termopar, temperatura de pico $\sim 810^{\circ} \mathrm{C}$.
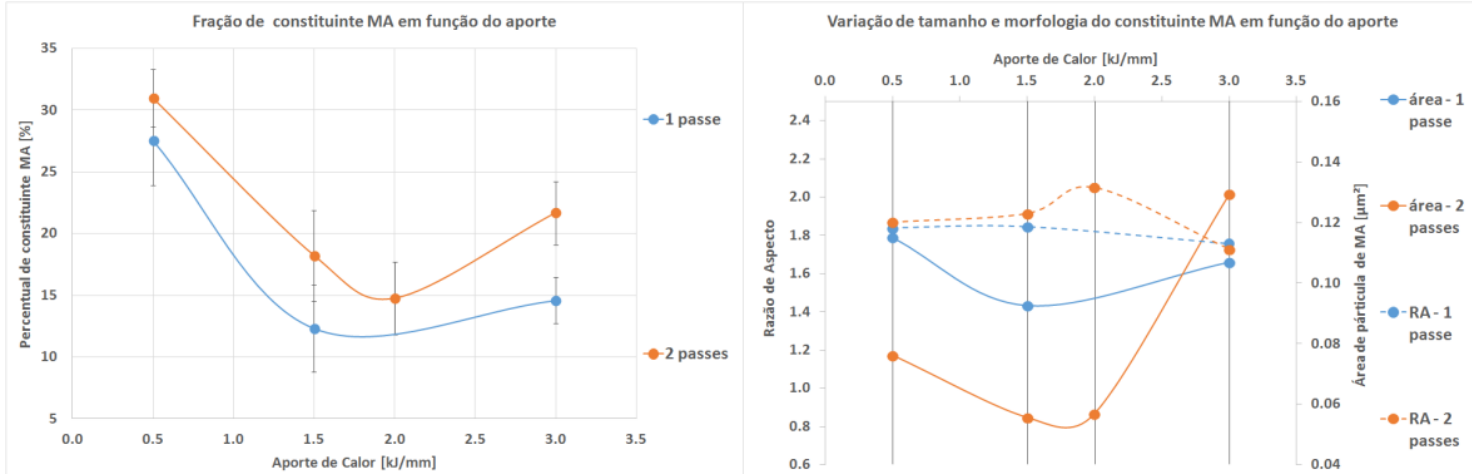

Figura 10. Variação da quantidade e forma do constituinte MA, para condição de passe único e duplo, em função do aporte de calor. À esquerda: variação de fração total de MA. À direita: variação da mediana da área individual de cada partícula e razão de aspecto em função do aporte.

\section{CONCLUSÃO}

1. Os extremos inferior e superior de aporte de calor resultaram na maior fração de MA, com ilhas mais grosseiras e massivas. Este fato pode indicar uma competição de dois fatores: tempo a altas temperaturas para segregação de carbono para austenita e taxa de resfriamento suficiente para reter e/ou temperar a austenita. Efeito mais marcante para condição de passe duplo.

2. Foi observada uma mudança brusca na morfologia do constituinte MA para uma variação de pouco mais de $30^{\circ} \mathrm{C}$ na temperatura de pico intercrítico, comprovando indicações da bibliografia e atentando ao perigo das regiões intercrítica de menor temperatura onde o MA se alastra conectado pelos contornos de austenita prévia.

3. O aporte de $2,0 \mathrm{~kJ} / \mathrm{mm}$ apresentou as melhores indicações para produção de uma junta de maior tenacidade: menor fração de MA, sem martensita e região de sobreposição de regiões GG-IC menos desfavorável.

4. Observou-se a importância do diagrama TRC no cálculo de previsões de fase e a dificuldade de modelar a formação do MA, dado o aspecto cinético da segregação de carbono, alteração de temperabilidade e regime intercrítico.

5. A alteração das temperaturas de início e fim da austenitização (A1 e A3) em função da taxa de aquecimento é significativa e deve ser considerada nas simulações de soldagem, considerando inclusive que parâmetros como aporte de calor, preaquecimento e velocidade de soldagem modificam a taxa de 
aquecimento e, portanto, podem alterar o regime de temperaturas da transformação da austenita.

\section{Agradecimentos}

Os autores agradecem à Tenaris (Brasil), pelo fornecimento de tubos e suporte financeiro, a Petrobras, pela cooperação técnica na pesquisa, em especial aos engenheiros Leonardo da Paixão Carvalho e Gilmar Zacca Batista, ao Senai CSTRJ, pela execução dos ensaios em Glleble, e ao Laboratório Nacional de Tecnologia da Soldagem (LNTSSold/Coppe/UFRJ), por sua colaboração.

\section{REFERÊNCIAS}

1 Zhuang C, Li N, Wang S, Lin W, Ren J. Microstructure and properties of heat-affected zones in X100 steel grade line pipes. Pressure Vessels and Piping Conference. 2009;6:1311-1319.

2 Lambert A, Drillet J, Gourgues AF, Sturel T, Pineau A. Microstructure of martensiteaustenite constituents in heat affected zones of high strength low alloy steel welds in relation to toughness properties. Science and technology of welding and joining. 2000;5(3):168-73.

3 Moeinifar S, Kokabi AH, Hosseini HM. Influence of peak temperature during simulation and real thermal cycles on microstructure and fracture properties of the reheated zones. Materials \& design. 2010;31(6):2948-55.

4 Davis CL, King JE. Cleavage initiation in the intercritically reheated coarse-grained heat-affected zone: Part I. Fractographic evidence. Metallurgical and materials transactions A. 1994;25(3):563-73.

5 Bhadeshia HKDH. About calculating the characteristics of the martensite-austenite constituent. Proceedings of International Seminar on Welding of High Strength Pipeline Steels. 2013;1:99-106.

6 Matsuda F, Fukada Y, Okada H, Shiga C, Ikeuchi K, Horii Y, Shiwaku T, Suzuki S. Review of mechanical and metallurgical investigations of martensite-austenite constituent in welded joints in Japan. Welding in the World/Le Soudage dans le Monde. 1996;3(37):134-54.

7 Li Y, Baker TN. Effect of morphology of martensite-austenite phase on fracture of weld heat affected zone in vanadium and niobium microalloyed steels. Materials Science and Technology. 2010;26(9):1029-40.

8 Li X, Fan Y, Ma X, Subramanian SV, Shang C. Influence of martensite-austenite constituents formed at different intercritical temperatures on toughness. Materials \& Design. 2015;67:457-63.

9 Li X, Ma X, Subramanian SV, Shang C, Misra RD. Influence of prior austenite grain size on martensite-austenite constituent and toughness in the heat affected zone of $700 \mathrm{MPa}$ high strength linepipe steel. Materials Science and Engineering: A. 2014;616:141-147.

10 Sysweld 2016: Reference Manual. ESI Group. 2016.

11 Liu Y, Yang LQ, Feng B, Bai SW, Xu CX. Physical simulation on microstructure and properties for weld HAZ of X100 pipeline steel. Materials Science Forum. 2013;762:556-561.

12 Fukuoka T. Analysis of Cooling Process of Arc Welding-Cooling Cycle at the Ends of Weld Bead. Marine Engineering Society in Japan. 1992;21(2):61-8. 